\title{
Directed self-assembly of field-responsive fluids in confined geometries $\dagger$
}

\author{
Ramin Haghgooie $^{* a}$ and Patrick S. Doyle ${ }^{* b}$ \\ Received 30th April 2008, Accepted 19th June 2008 \\ First published as an Advance Article on the web 6th August 2008 \\ DOI: $10.1039 / b 807389 a$
}

\begin{abstract}
We present an investigation into the important physical principles associated with the self-assembly of magnetorheological (MR) fluids in the microfluidic setting. We concentrate on the role of channel topology in influencing the resulting microstructure. In particular, using Brownian dynamics simulations, we show how a variety of geometrically-simple confining microchannels can be used to strongly control the lattice type and orientation in self-assembled MR fluids. Additionally, we demonstrate how topographical features can be used to dictate the order (or disorder) of the steadystate structure. We highlight the similarities and differences between our three-dimensional microchannel system and the structures and dynamics observed in two-dimensional confined systems. Furthermore, we present an example of the introduction of local magnetic field inhomogeneity and its strong influence over the resulting self-assembled MR fluid structure.
\end{abstract}

\section{Introduction}

In recent years, self-assembled systems have garnered much attention due to their potential for creating order on very small scales as well as their obvious analogy to biological systems. ${ }^{1-4}$ When considering these systems, one must be aware of the ever present issue of boundary effects which can strongly influence the behavior and structure of the self-assembled system. ${ }^{5-7}$ In many cases, the boundary effects are caused by a confined system in which the assembly is taking place. For instance, colloidal assembly in microfluidic devices has been shown to be a promising route for manufacturing micron, or sub-micron, structured systems. ${ }^{8-13}$ Furthermore, the study of confined self-assembly has revealed many interesting phenomena which depend on the nature of the confinement. ${ }^{14-21}$ In the current study we will investigate the self-assembly of magnetorheological (MR) fluids in microfluidic confinement and the important role of channel topology.

We will focus on MR colloids in the presence of a uniform external magnetic field and in the absence of flow. When confined in the field direction, the colloids in a MR fluid self-assemble into column-like structures aligned with the field and arranged in a hexagonal pattern. ${ }^{15,17}$ If the confining planes are separated by a single colloid diameter by definition the system is two-dimensional (2D) and the structure is determined by only a single degree of freedom (a convolution of the volume fraction of colloids and the strength of the external field). ${ }^{22}$ Increasing the separation of the confining planes to create a 3D system yields a rich phase behavior including many different structural configurations. ${ }^{16,23}$ Alternatively, introducing lateral confinement into such systems can alter the self-assembled structures and lead to interesting structural properties. Self-assembly of

${ }^{a}$ Center for Engineering in Medicine, Massachusetts General Hospital, Boston, USA. E-mail: rhaghgooie@partners.org

${ }^{b}$ Department of Chemical Engineering, Massachusetts Institute of Technology, Cambridge, USA.E-mail: pdoyle@mit.edu

$\dagger$ This paper is part of a Soft Matter theme issue on Self-Assembly. Guest editor: Bartosz Grzybowski.
MR fluids in laterally confined systems has predominately been studied in 2D where the lateral confinement is in the form of closed cavities ${ }^{18,19}$ or open channels. ${ }^{7,20,24}$ In $2 \mathrm{D}$ closed cavities, it has been shown that the confining boundaries influence the lattice type formed by a finite number of MR colloids, ${ }^{18,19}$ while in $2 \mathrm{D}$ channels we have previously shown that the channel width plays a crucial role in determining how ordered the hexagonal colloidal structure can become. ${ }^{7,20,24}$ Additionally, in both cases, the presence of parallel non-magnetic walls induces extreme localization of colloids along those walls.

In this work we explore the self-assembly of MR fluids in 3D microchannels where the height in the field direction is varied down the length of the channel. A schematic of the general geometry is shown in Fig. 1. This geometry is easily created in the microfluidic community using a simple two-step soft lithographic process. ${ }^{25}$ Among our primary interests is to control the type of lattice (orientation, spacing, and order/disorder) formed by the MR colloids in these systems without altering their utility (i.e. still enabling throughput of fluid and molecules). Such control
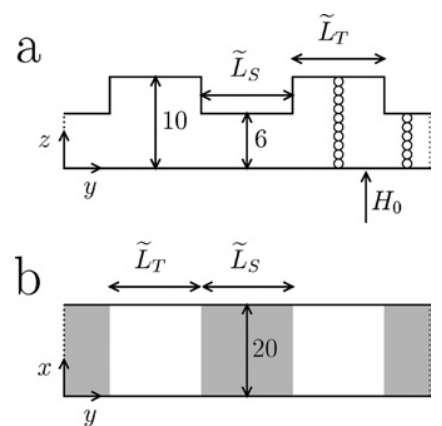

Fig. 1 A schematic illustrating the geometry of the stepped channels. (a) Side view of the confining geometry which is periodic in the $y$ direction. The channel has alternating regions of dimensionless height 10 and 6 with lengths $\tilde{L}_{\mathrm{T}}$ and $\tilde{L}_{\mathrm{S}}$ respectively. (b) Top view of the confining geometry showing the dimensionless width of 20 . The short and tall regions are indicated by shaded and non-shaded regions respectively. The uniform magnetic field is oriented in the $z$ direction. 
will be critical for applications such as bio-molecule separations that rely upon the microstructure in the system that acts like a sieve. ${ }^{9,11,26,27}$

It is important to contrast the present system with the previous work of our group and others in 2D systems. In 2D cavities, the system boundaries define a finite closed area in which colloidal assembly takes place. ${ }^{\mathbf{1 8 , 1 9}}$ While this is a model system for studying the effects of closed boundaries upon self-assembly it is not practical for microfluidic applications. In 2D channels, the system remains open along one dimension and the colloids selfassemble between the two parallel closed boundaries defining the channel walls. ${ }^{7,20,24}$ In this case, the walls are the only geometrical feature that can induce boundary effects upon the system. In the present study, the channel side walls exert boundary effects upon the system in much the same way as $2 \mathrm{D}$ channels but the addition of non-uniform topography along the other confining direction creates pseudo-boundaries that influence structure formation as well. Further, we consider 3D systems that are not only more realistic for applications, but also fundamentally different than purely $2 \mathrm{D}$ monolayers. ${ }^{16,23}$ The central question remains open as to how topographical features in microchannels can influence the structure of self-assembled MR fluids.

\section{Simulation details}

When subjected to a uniform external magnetic field, the colloids in a MR fluid acquire dipole moments whose interactions can be modeled by the energy:

$$
\tilde{U}_{i j}\left(\tilde{r}_{i j}, \theta_{i j}\right)=\frac{1}{2} \lambda\left(\frac{1-3 \cos ^{2} \theta_{i j}}{\tilde{r}_{i j}^{3}}\right)
$$

The interaction energy between two colloids $\tilde{U}_{i j}$ is made dimensionless with the thermal energy $\left(\tilde{U}_{i j}=U_{i j} / k_{\mathrm{B}} T\right)$ and the separation distance between the centers of colloids $i$ and $j$ is scaled by the colloid diameter $\left(\tilde{r}_{i j}=r_{i j} / d\right)$. The parameter $\theta_{i j}$ is the angle between the vector $\boldsymbol{r}_{i j}$ and the external field vector. This interaction energy neglects the effects of mutual induction that can be present in real MR fluids but it has been successfully used to quantitatively predict the behavior of MR fluids. ${ }^{28}$ The dimensionless magnetic field strength $\lambda$ is defined as:

$$
\lambda \equiv-\tilde{U}_{i j}(1,0)=\frac{\pi \mu_{0} d^{3} \chi^{2} H_{0}{ }^{2}}{72 k_{\mathrm{B}} T}
$$

where $\mu_{0}$ is the permeability of free space, $\chi$ is the effective magnetic susceptibility of an individual MR colloid, and $H_{0}$ is the magnitude of the external magnetic field. The dimensionless field strength is the ratio of the maximum magnitude interaction energy to the thermal energy in the system. ${ }^{17}$ When $\lambda \gg 1$ the MR fluid will self-assemble into structures aligned with the external magnetic field.

Brownian dynamics simulations were used to model the motion of the MR colloids as previously described in detail. ${ }^{15}$ Briefly, the equation of motion for the colloids was given by:

$$
\mathrm{d} \mathbf{r}_{i}(t) \simeq \frac{1}{\zeta} \mathbf{F}_{\mathrm{D}, i}(t) \mathrm{d} t+\frac{1}{\zeta} \mathbf{F}_{\mathrm{B}, i}(t) \mathrm{d} t
$$

where the inertia of the colloids is neglected. ${ }^{29}$ The term $\mathbf{F}_{\mathrm{D}, i}$ represents all of the deterministic forces acting upon colloid $i$ (due solely to magnetic interactions) and $\zeta$ is the drag coefficient on a single colloid. $\mathbf{F}_{\mathrm{B}, i}$ is a stochastic term used to model the Brownian force acting on the colloid. Hydrodynamic interactions between the colloids were neglected for simplicity.

Eqn 3 was integrated forward in time using a simple Euler integration scheme. At the end of each time-step, hard sphere overlaps were treated by displacing overlapped colloids along the line connecting their centers until they are just contacting. ${ }^{30}$ This procedure was performed for all overlaps between two colloids and between colloids and hard walls ${ }^{15,20}$ and was iterated until all overlaps in the system were removed.

The external field was directed along the $z$-axis and hard wall boundary conditions were imposed in the $x$ and $z$ directions. The system was periodic in the $y$ direction thus creating a channel-like geometry along the $y$-axis. The volume fraction of MR colloids was held constant at $\phi=0.03$ and the channel width in the $x$ direction was fixed at a dimensionless length of 20 . The dimensionless length of the simulation box in the $y$ direction ranged from 80 to 180 depending upon the geometry in the $z$ direction and the total number of colloids in the simulations therefore ranged from 825 to 1650 . A dimensionless time-step of $\Delta \tilde{t}=1 \times$ $10^{-4}$ was used with time made dimensionless as $\tilde{t}=t\left(k_{\mathrm{B}} T\right) /\left(\zeta d^{2}\right)$, where $\left(\zeta d^{2}\right) /\left(k_{\mathrm{B}} T\right)$ is the time necessary for a MR colloid to freely diffuse a distance equal to its diameter. A dimensionless cutoff of 20 was used for the dipole-dipole interaction along with a linked list binning algorithm ${ }^{31}$ where the bin sizes were slightly larger than the cutoff value. The simulations were started from a random configuration and the external magnetic field was ramped continuously from $\lambda=0$ to $\lambda=100$ over a dimensionless time of 50 after which it was held constant at $\lambda=100$. All of the simulations were determined to be converged in system size, time-step, cutoff for the magnetic interactions, and ramp time for the external field.

\section{Results}

Snapshots of the structure that forms in three representative channels are shown in Fig. 2 . The step lengths $\left(\tilde{L}_{\mathrm{S}}, \tilde{L}_{\mathrm{T}}\right)$ are $(5,10)$, $(10,20)$ and $(30,5)$ respectively for the three channels a, b, and c. As expected, the clusters are aligned in the field direction into columns of magnetic colloids. There are several interesting features of the structure in these three channels. In the configuration snapshots (top row) the alignment of the clusters along the channel wall is evident and is consistent with observations of $2 \mathrm{D}$ channel systems. ${ }^{7}$ Interestingly, in Fig. 2a, clusters appear to be excluded from the short regions (shaded areas) and in Fig. $2 b$

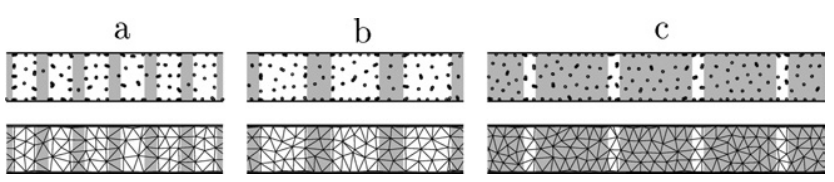

Fig. 2 The top row contains snapshots of the configuration of colloids in the channel for (a) $\tilde{L}_{\mathrm{S}}=5$ and $\tilde{L}_{\mathrm{T}}=10$, (b) $\tilde{L}_{\mathrm{S}}=10$ and $\tilde{L}_{\mathrm{T}}=20$, and (c) $\tilde{L}_{\mathrm{S}}=30$ and $\tilde{L}_{\mathrm{T}}=5$ respectively. The bottom row contains Delaunay triangulation plots of nearest-neighbor pairs of clusters for the same three channels. The short and tall regions are indicated by shaded and nonshaded regions respectively. The uniform magnetic field is oriented normal to the page. 
they appear to consistently have room for one line parallel to the step. The exclusion, or partial exclusion, of clusters from the short regions is surprising. Considering the ability of the colloids to freely migrate down the channel during the assembly process, a constant density of clusters may be expected. However, these features may be explained when we take into account the theoretical spacing between colloids in an equivalent $2 \mathrm{D}$ system. We have previously defined this spacing as: ${ }^{15}$

$$
\langle\tilde{s}\rangle_{2 \mathrm{D}} \equiv \sqrt{\frac{\pi}{3 \phi \sqrt{3}}}
$$

and for the current study $\langle\tilde{s}\rangle_{2 \mathrm{D}}=4.49$. Therefore, in the channel shown in Fig. 2a, the short sections are equivalent to $\langle\tilde{s}\rangle_{2 \mathrm{D}}$ indicating that there is not room for clusters to form within these sections. Likewise, in the channel shown in Fig. $2 b$ the short sections have a length that is equivalent to $2\langle\tilde{s}\rangle_{2 \mathrm{D}}$ and thus there is only room for one row within these sections.

Aside from the equivalence of the length scales of cluster spacing and step length, there is one other feature of the structure in these systems that has not been previously observed. In Fig. 2 the clusters are also seen to align along the steps in the channel (edges of the shaded regions in Fig. 2) creating repulsive pseudoboundaries for the short sections and giving rise to the behavior observed in Fig. 2a and b. While the pseudo-boundaries strongly affect the structure, they do not change the open nature of the system. These lines of clusters impose a secondary orientation upon the lattice. This secondary orientation is more easily observed in the triangulations shown in the bottom row of Fig. 2. Each line segment in these figures connects two nearest-neighbor clusters as determined by Delaunay triangulation. ${ }^{15}$ In Fig. 2a and $b$ the emergence of a square lattice can be observed in the triangulation plots while in Fig. 2c, the structure in the system remains predominately hexagonal with only small regions that appear square.

In order to further quantify the type of structure present in the channels, we determined the probability density for two nearestneighbor clusters to be oriented at a particular angle $\left(\theta_{n}\right)$ with respect to the $y$-axis (or channel walls). These probability distributions are shown in Fig. 3 for the three channels discussed above. Because of the symmetry of our system, we plot $\theta_{n}$ for $0 \leq$ $\theta_{n}<180$ and $\theta_{n}-180$ for $180 \leq \theta_{n}<360$. A perfectly aligned hexagonal structure within the channel would have peaks at the

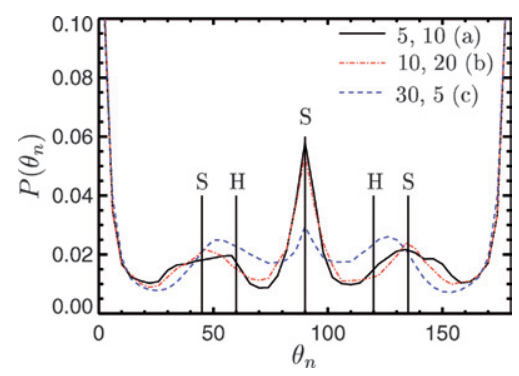

Fig. 3 Angles between nearest-neighbor clusters with reference to the $y$-axis (channel walls). Three different channel geometries are shown: $\tilde{L}_{\mathrm{S}}$ $=5, \tilde{L}_{\mathrm{T}}=10$ (solid black line), $\tilde{L}_{\mathrm{S}}=10, \tilde{L}_{\mathrm{T}}=20$ (dash-dot red line), and $\tilde{L}_{\mathrm{S}}=30, \tilde{L}_{\mathrm{T}}=5$ (dashed blue line). The vertical lines mark the theoretical peak locations for a perfect square lattice (S) and a perfect hexagonal lattice $(\mathrm{H})$ oriented in the channel direction. locations indicated by the letter " $\mathrm{H}$ " in Fig. 3 while a perfectly aligned square structure would have peaks at the locations indicated by the letter "S". From the distributions in Fig. 3 we observe that two of the channels appear to have square symmetry while the third predominately retains its hexagonal symmetry. Channels $\mathrm{a}$ and $\mathrm{b}$ exhibit strong square symmetry with large peaks at $\theta_{n}=0,90$, and 180 and secondary peaks at $\theta_{n}=45$, and 135. Channel $\mathrm{c}$ exhibits a drastically different structure with peaks closer to $\theta_{n}=0,60,120$ and 180 indicative of a hexagonal structure. Channel c still exhibits a peak at $\theta_{n}=90$ as is expected due to the alignment of clusters along the steps, but the magnitude of that peak is small in comparison with channels a and $b$.

In order to achieve a broader picture of the effects of channel geometry upon the lattice structure in these systems, we define the local bond-orientational order parameters $\left\langle\left|\Psi_{6}\right|\right\rangle$ and $\left\langle\left|\Psi_{4}\right|\right\rangle$. We have used $\left\langle\left|\Psi_{6}\right|\right\rangle$ previously to characterize the structure in 2D dipole systems. ${ }^{7,20}$ The order parameters are defined as:

$$
\begin{aligned}
& \left\langle\left|\Psi_{6}\right|\right\rangle \equiv \frac{1}{N} \sum^{N}\left|\frac{1}{m} \sum_{k=1}^{m}{\exp i 6 \theta_{k} \mid}\right| \\
& \left\langle\left|\Psi_{4}\right|\right\rangle \equiv \frac{1}{N} \sum^{N}\left|\frac{1}{m} \sum_{k=1}^{m}{\exp i 8 \theta_{k} \mid}^{m}\right|
\end{aligned}
$$

where $m$ is the number of nearest-neighbors for a given cluster and $\theta_{k}$ is the angle between the vector connecting the cluster and its $k$ th nearest-neighbor and an arbitrary reference axis. If the lattice is perfectly hexagonal then $\left\langle\left|\Psi_{6}\right|\right\rangle=1$, if it is perfectly square then $\left\langle\left|\Psi_{4}\right|\right\rangle=1$, and if it is totally disordered then both order parameters approach zero.

In Fig. 4 we present state diagrams indicating the symmetry of the structure that forms in these channel systems. In Fig. 4a and c, each circle symbol represents a simulation result for a particular geometry. The points along $\tilde{L}_{\mathrm{S}}=\infty$ and $\tilde{L}_{\mathrm{T}}=\infty$ indicate the value of $\left\langle\left|\Psi_{6}\right|\right\rangle$ or $\left\langle\left|\Psi_{4}\right|\right\rangle$ for purely rectangular channels of dimensionless height 6 and 10 respectively. In order to more clearly observe the trends in the state diagram we present in Fig. $4 \mathrm{~b}$ and $\mathrm{d}$ an interpolated continuous contour plot for the same data. The most obvious feature of the data is the large region in the lower left corner where the structure has weak hexagonal symmetry. Clearly, the region in Fig. 4b that is least hexagonal is the same region in Fig. 4d that exhibits the most square symmetry. As one or both of the step lengths increases, the structure appears to become more hexagonal. However, even at dimensionless step lengths of 30 , the system is far from the infinite limit. This is consistent with results for $2 \mathrm{D}$ channel confinement $^{7,20}$ where a length (or channel width) of 30 colloid diameters is still strongly influenced by the presence of the confining boundaries. In contrast to $2 \mathrm{D}$ channels however, in this case the important features are not confining boundaries but rather topographical features. Additionally, in Fig. 4d at larger step lengths, the structure retains much more square symmetry than the constant height case. There are many other subtle features of the state diagram for this system most likely due to the confluence of a number of complicating effects. These particular channel systems are subject to the subtleties of 2D channel confinement, ${ }^{7,20}$ thin-slit confinement, ${ }^{15,23}$ and confinement in pseudo-cavities ${ }^{19}$ all of which contribute to the complexity of the structure observed. 

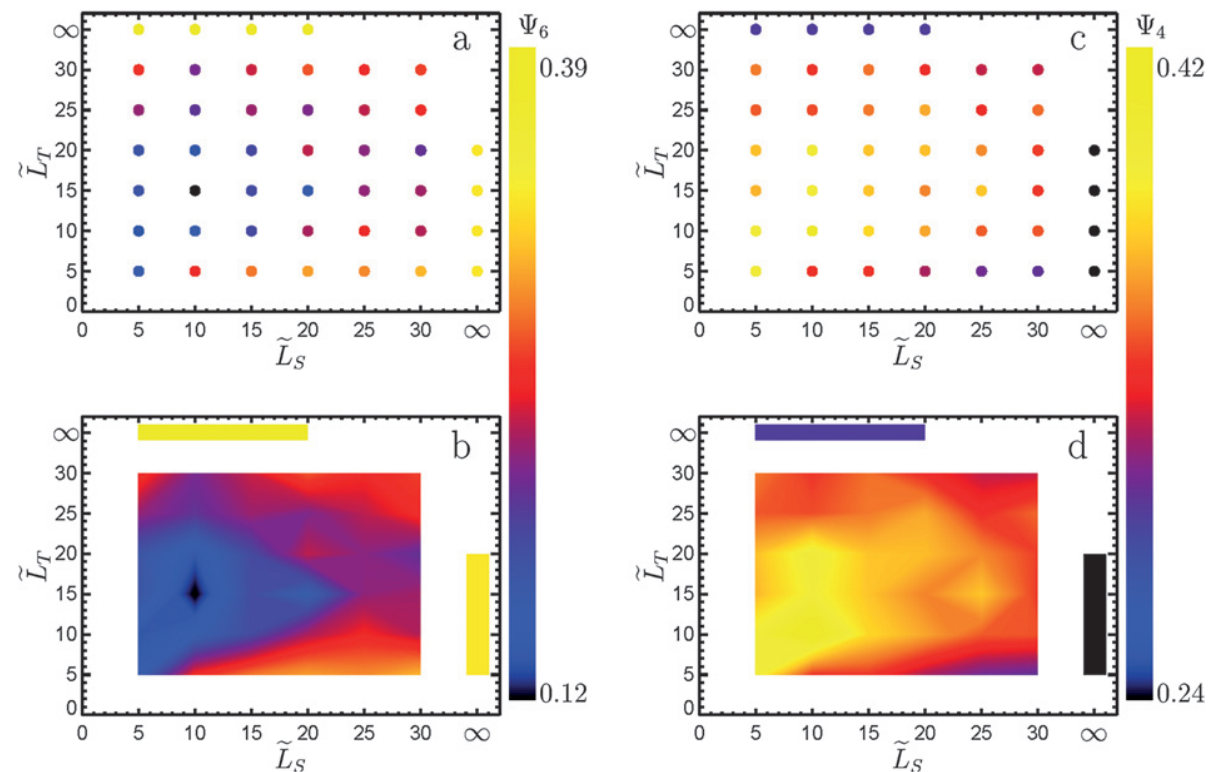

Fig. 4 State diagram of the order parameters $\Psi_{6}(\mathrm{a}, \mathrm{b})$ and $\Psi_{4}(\mathrm{c}, \mathrm{d})$ as a function of $\tilde{L}_{\mathrm{S}}$ and $\tilde{L}_{\mathrm{T}}$. (a and c) Individual simulation data points. (b and d) Interpolated contour plot showing regions of strong and weak hexagonal (b) and square (d) structure. The points at $\infty$ on either axis represent the structure in a straight rectangular channel of height 6 in the case of $\tilde{L}_{\mathrm{S}}=\infty$ and height 10 in the case of $\tilde{L}_{\mathrm{T}}=\infty$.

Within the regions of pseudo-confinement defined by the tall sections of the channel, we not only observe structure reminiscent of $2 \mathrm{D}$ cavity systems ${ }^{19}$ but we also observe some very interesting dynamics. In Fig. 5 we present the trajectories of clusters in a small section of the channel for the same three cases (a, b, and c) as presented above. The trajectories are over a dimensionless time of 100 during which the cluster positions were sampled 1000 times. The first feature to note in Fig. 5 is the behavior of the clusters along the walls and along the steps. Both along the walls and along the steps, the clusters are closely constrained in the direction normal to the confining boundary and are more free to fluctuate along the boundary. This is especially evident in Fig. 5b. While this behavior is expected along closed boundaries, it is surprising to note that the effect is just as pronounced along the steps which are open edges. Additionally, in the corners of the tall regions, the clusters are constrained in both directions and barely migrate at all. This feature can be observed in Fig. $5 \mathrm{c}$ where, at the top of the figure, there is a single cluster in the tall region that fluctuates along half the length of the wall while the two clusters at the bottom of the
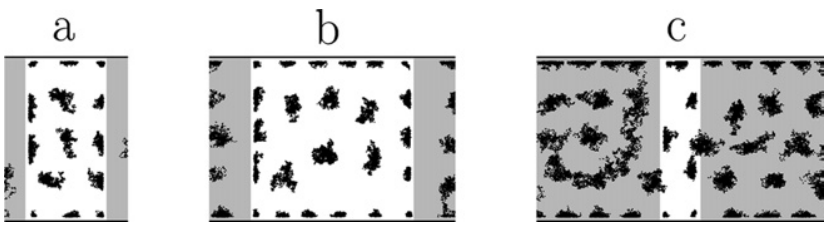

Fig. 5 Traces of cluster positions over a dimensionless time of 100 for one periodic unit in channels with (a) $\tilde{L}_{\mathrm{S}}=5$ and $\tilde{L}_{\mathrm{T}}=10$, (b) $\tilde{L}_{\mathrm{S}}=10$ and $\tilde{L}_{\mathrm{T}}=20$, and (c) $\tilde{L}_{\mathrm{S}}=30$ and $\tilde{L}_{\mathrm{T}}=5$ respectively. The cluster positions were sampled 1000 times over the course of the trajectories. The short and tall regions are indicated by shaded and non-shaded regions respectively. The uniform magnetic field is oriented normal to the page. figure in the corners of the tall region do not leave the immediate vicinity of the corners.

A second feature that is evident is the absence of clusters in the short region in Fig. 5a, the alignment of clusters down the center of the short region in Fig. 5b, and the fact that in Fig. 5c the clusters in the short region arrange in a hexagonal pattern that is not perturbed by the presence of the tall region. In all three cases, the clusters in the bulk (i.e. clusters not along the walls or steps) appear to be able to fluctuate freely in any direction around their lattice position.

Perhaps the most striking features of the trajectories presented are the rectangular sub-systems that catch the eye in Fig. 5a and b. In the tall region, the clusters arrange themselves into a rectangular cage in which the remaining clusters assemble into a local lattice. This behavior is analogous to the behavior observed in 2D cavity systems. ${ }^{18,32}$ However, in this case, there exist no walls parallel to the $x$-axis and the entire channel system is open in that direction. This is an important point because even though we have not physically confined the MR colloids, they assemble into a structure that mimics that of a confined system.

Thus far we have demonstrated the ability to generate, in open systems, structures that mimic closed cavity systems. Additionally, by imposing pseudo-confinement with stepped regions in the channel we demonstrated the ability to change the equilibrium lattice symmetry. We will now explore methods to affect lattice orientation within the channel by allowing the orientation of the stepped regions to vary. These microfluidic geometries are also simple to manufacture with a two-step lithographic process. ${ }^{25}$ The step-orientation of the systems presented above was $\theta_{s}=90$ with respect to the $y$-axis. In Fig. 6 we present configuration snapshots for three different channels (a, b, and c) with step angles of $\theta_{s}=60,45$, and 30 respectively. The length of the short sections (shaded areas) is $\tilde{L}_{\mathrm{S}}=10$ and the tall sections have a length of $\tilde{L}_{\mathrm{T}}=20$. Even when the angle of the step is varied, the 


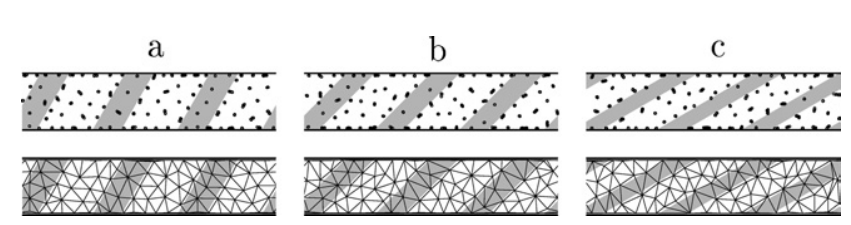

Fig. 6 The top row contains snapshots of the configuration of colloids in the channel for $\tilde{L}_{\mathrm{S}}=10$ and $\tilde{L}_{\mathrm{T}}=20$ and (a) $\theta_{s}=60$, (b) $\theta_{s}=45$, (c) $\theta_{s}=$ 30 respectively. The bottom row contains Delaunay triangulation plots of nearest-neighbor pairs of clusters for the same three channels. The short and tall regions are indicated by shaded and non-shaded regions respectively. The uniform magnetic field is oriented normal to the page.

alignment of clusters along the step in the tall sections is still evident in the snapshots along the top row of Fig 6. Additionally, as the angle is decreased, clusters begin to become excluded from the short sections of the channel ( $c f$. Fig. 6a and c) even though their relative fraction of the total channel volume remains constant. Most notably, because of the templating due to the presence of the steps, in some instances, the lattice becomes more aligned with the channel walls as in Fig. 6a and in other instances it becomes less aligned with the channel walls as in Fig. 6c.

In order to see more clearly the effects of the step angle on the lattice type in these channels, we present in Fig. 7 the dependence of the two order parameters $\left\langle\left|\Psi_{6}\right|\right\rangle$ and $\left\langle\left|\Psi_{4}\right|\right\rangle$ upon $\theta_{s}$. When the step angle is 90, the case in Fig. 2 to 5, the lattice type is much more square than hexagonal as we have already shown. As $\theta_{s}$ is decreased however, there is a dramatic effect upon the structure in the system. As $\theta_{s}$ approaches 60, the structure becomes much more hexagonal than square (this can also be seen in the bottom row of Fig. 6a). As $\theta_{s}$ goes to 45 the system appears to be equally structured in terms of both order parameters and it is not immediately apparent which type of structure dominates. Upon careful observation of the Delaunay triangulation for this channel in Fig. 6b, we observe an equivalent number of square sites and hexagonal sites. Additionally, there are a couple clusters with up to nine neighbors, disrupting both square and hexagonal lattices. As $\theta_{s}$ is further decreased to a value of 30 , the structure in the system does not resemble either type of lattice. In fact, it appears that order has been almost completely disrupted in this system. Fig. 6 and 7 serve to illustrate, to our knowledge, the first instance of geometric topography influencing the structure and lattice type of self-assembled field-responsive fluids in 3D systems. Rather than confining the colloids to a single cavity which defines the entire system, the geometry in this case simply

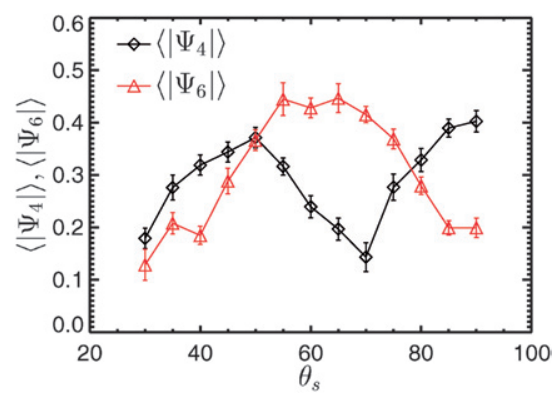

Fig. 7 Values of the order parameters $\left\langle\left|\Psi_{4}\right|\right\rangle$ (black diamonds) and $\left\langle\left|\Psi_{6}\right|\right\rangle$ (red triangles) as a function of the step angle $\left(\theta_{s}\right)$ in a channel with $\tilde{L}_{\mathrm{S}}=10$ and $\tilde{L}_{\mathrm{T}}=20$.

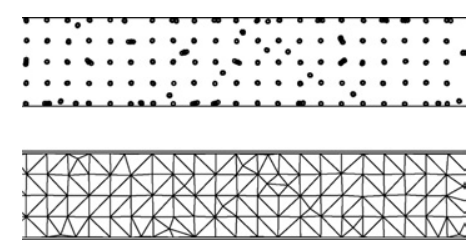

Fig. 8 The top image is a snapshot of the colloid positions in a templated channel with dimensionless height of 6 and dimensionless width of 20. A single layer of MR colloids (not shown) is fixed in a square lattice with dimensionless spacing of 4.75 on the bottom of the channel. The bottom image is a Delaunay triangulation plot of nearest-neighbor pairs of clusters for the same channel. The uniform magnetic field is oriented normal to the page.

serves to template the structure that forms. Such epitaxy effects have been used to successfully grow single colloidal crystals upon a topographically patterned substrate ${ }^{33}$ but have never been demonstrated for field-responsive fluids, in which colloids interact anisotropically and self-assembly is a fundamentally different process.

A very effective, albeit more complex, alternative to geometric control of self-assembled structure is to introduce local inhomogeneities in the magnetic field. In the microfluidic setting, this can be accomplished by patterning magnetic materials at discrete positions within, or near, the fluidic channels. ${ }^{12,34,27}$ When a uniform external magnetic field is applied to the system, the magnetic material generates its own non-uniform field that influences the motion of the MR colloids. As an alternative to patterning continuous regions of magnetic materials, one may pattern discrete colloids to achieve the same effects. ${ }^{35}$ While this approach has the potential to be extremely effective for influencing self-assembled structure of MR fluids, it requires rather complex manufacturing techniques which limit its usefulness. Nonetheless, we also present the power of this approach with a simple example. Fig. 8 is a snapshot of the structure that forms in a templated channel of dimensionless width 20 and dimensionless height 6 . In this example, a single layer of MR colloids is immobilized on the bottom surface of the channel in a square lattice with a dimensionless spacing of 4.75 between colloids. This spacing is of the same magnitude as the natural spacing $\left(\left\langle\tilde{S}_{2 \mathrm{D}}\right\rangle\right)$ of the hexagonal lattice formed in this system as presented above. The snapshot in the top of Fig. 8 shows the locations of the remaining colloids in this system. It is clear in Fig. 8 that the templated pattern completely determines the lattice that forms in this case. In both the snapshot and the triangulation plot, the lattice appears square. The order parameters for the self-assembled structure in this system are $\left\langle\left|\Psi_{6}\right|\right\rangle=0.14$ and $\left\langle\left|\Psi_{4}\right|\right\rangle=0.80$ respectively and confirm that the lattice type is square. As mentioned, the microchannel fabrication involved in creating the template pattern shown in Fig. 8 is more difficult than the creation of stepped microchannels. However, technologies are currently being developed that allow for such templates to be created more easily within a microfluidic setting ${ }^{12,35}$

\section{Summary}

We have outlined several design strategies to create useful spatial motifs of MR colloids in microfluidic channels. We show that 
channels with a stepped-height topography can alter the lattice type, orientation, and order. In contrast to $2 \mathrm{D}$ monolayer systems, in our system there are no closed cavities, but instead pseudo-cavities created by the topography of one confining boundary. The flexibility of this simple method will have tremendous impact upon DNA separation devices that rely upon the structure of self-assembled MR fluids ${ }^{9,11}$ since the orientation and order of the lattice has been shown to be an important consideration. ${ }^{36}$

While the present study contains only simulation results, the channel motifs presented here have been previously realized ${ }^{25}$ and are thus experimentally accessible. Such geometries have never been explored for directing self-assembly of field-responsive fluids and we have presented only a single, yet informative example of how they might be utilized. In general, the use of topographically varying confinement to control the 3D selfassembly of MR fluids has not been explored. Thus, there remain several interesting open questions as to the breadth of possibilities along the lines of the work presented here. For instance, we have presented a case where the topographical features can easily be considered units of a larger lattice. However, if the features are incompatible with any lattice, such as circular indentations, one may expect completely different behavior. Further, the addition of another driving force, such as flow, will create rich anisotropic colloid dynamics which could be exploited for sorting applications. In the future, we will address this and many other questions about how one may use topography to direct the selfassembly of field-responsive fluids.

\section{Acknowledgements}

P. Doyle gratefully acknowledges the support of NSF NIRT Grant No. CTS-0304128 and Singapore-MIT Alliance (SMA-II, CPE Program) for this project. R. Haghgooie gratefully acknowledges the MIT-MGH Postdoctoral Fellowship in Translational Research for financial support.

\section{References}

1 K. Channon and C. E. MacPhee, Soft Matter, 2008, 4(4), 647-652.

2 R. M. Capito, H. S. Azevedo, Y. S. Velichko, A. Mata and S. I. Stupp, Science, 2008, 319(5871), 1812-1816.

3 B. Zhao, H. Hu, S. K. Mandal and R. C. Haddon, Chem. Mater., $2005,17,3235$.

4 B. A. Grzybowski, M. Radkowski, C. J. Campbell, J. N. Lee and G. M. Whitesides, Appl. Phys. Lett., 2004, 84(10), 1798-1800.
5 A. Blanco, E. Chomski, S. Grabtchak, M. Ibisate, S. John, S. W. Leonard, C. Lopez, F. Meseguer, H. Miguez, J. P. Mondia, G. A. Ozin, O. Toader and H. M. van Driel, Nature, 2000, 405, 437.

6 R. A. Segalman, A. Hexemer and E. J. Kramer, Macromolecules, 2003, 36, 6831 .

7 R. Haghgooie and P. S. Doyle, Phys. Rev. E, 2004, 70, 061408.

8 G. H. Seong and R. M. Crooks, J. Am. Chem. Soc., 2002, 124, 1336013361.

9 P. S. Doyle, J. Bibette, A. Bancaud and J.-L. Viovy, Science, 2002, 295, 2237.

10 G. C. Randall and P. S. Doyle, Proc. Natl. Acad. Sci. U. S. A., 2005, 102(31), 10813.

11 N. Minc, P. Bokov, K. B. Zeldovich, C. Fütterer, J.-L. Viovy and K. D. Dorfman, Electrophoresis, 2005, 26, 362.

12 A.-E. Saliba, N. Minc, J.-L. Viovy, C. Gosse and C. Roblin, Houille Blanche, 2007, 6, 34-38.

13 E. M. Furst, C. Suzuki, M. Fermigier and A. P. Gast, Langmuir, 1998, 14, 7334 .

14 A. van Blaaderen, R. Ruel and P. Wiltzius, Nature, 1997, 385(6614), 321.

15 R. Haghgooie and P. S. Doyle, Phys. Rev. E, 2007, 75, 061406.

16 T. Ukai and T. Maekawa, Phys. Rev. E, 2004, 69, 032501.

17 J. Liu, E. M. Lawrence, A. Wu, M. L. Ivey, G. A. Flores, K. Javier, J. Bibette and J. Richard, Phys. Rev. Lett., 1995, 74(14), 2828-2831.

18 M. Kong, B. Partoens, A. Matulis and F. M. Peeters, Phys. Rev. E, 2004, 69, 036412.

19 R. Bubeck, S. Neser, C. Bechinger and P. Leiderer, Prog. Colloid Polym. Sci., 1998, 110, 41-45.

20 R. Haghgooie and P. S. Doyle, Phys. Rev. E, 2005, 72, 011405.

21 M. A. Hayes, N. A. Polson and A. A. Garcia, Langmuir, 2001, 17, 2866.

22 K. Zahn, R. Lenke and G. Maret, Phys. Rev. Lett., 1999, 82(13), $2721-2724$.

23 R. Haghgooie and P. S. Doyle, Europhys. Lett., 2007, 77, 18002.

24 R. Haghgooie, C. Li and P. S. Doyle, Langmuir, 2006, 22, 3601-3605.

25 A. D. Stroock, S. K. W. Dertinger, A. A. I. Mezić, H. A. Stone and G. M. Whitesides, Science, 2002, 295, 647-651.

26 M. Slovakova, N. Minc, Z. Bilkova, C. Smadja, W. Faigle, C. Futterer, Myriam. and J. L. Viovy, Lab Chip, 2005, 5, 935-942.

27 N. Pamme, Lab Chip, 2006, 6, 24-38.

28 M. Mohebi, N. Jamasbi and J. Liu, Phys. Rev. E, 1996, 54(5), 54075413.

29 H. C. Öttinger, Stochastic Processes in Polymeric Fluids: Tools and Examples for Developing Simulation Algorithms, Springer, Berlin, 1996.

30 D. Heyes and J. Melrose, J. Non-Newtonian Fluid Mech., 1993, 46, 128.

31 D. Frenkel and B. Smit, Understanding Molecular Simulation from Algorithms to Applications, MPG Books Ltd, Bodmin, Great Britain, 2nd edn, 2002.

32 R. Bubeck, C. Bechinger, S. Neser and P. Leiderer, Phys. Rev. Lett., 1999, 82(16), 3364.

33 K. H. Lin, J. C. Crocker, V. Prasad, A. Schofield, D. A. Weitz, T. C. Lubensky and A. G. Yodh, Phys. Rev. Lett., 2000, 85, 1770.

34 S. Bhat, J. Guez, T. P. Kurzweg, A. Guez and G. Friedman, IEEE Trans. Magn., 2007, 43(6), 2427-2429.

35 D. C. Pregibon, M. Toner and P. S. Doyle, Langmuir, 2006, 22, 5122.

36 A. Mohan and P. S. Doyle, Phys. Rev. E, 2007, 76(4), 040903. 